\title{
An Actuated Finger Exoskeleton for Hand Rehabilitation Following Stroke
}

\author{
T.T. Worsnopp, M.A. Peshkin, J.E. Colgate, and D.G. Kamper, Member, IEEE
}

\begin{abstract}
Chronic hand impairment is common following stroke. While mass practice of movement has shown promise for rehabilitation, initial impairment of the hand may be too severe to permit even approximations of the desired movement. To facilitate movement, especially of pinch, we are building an exoskeleton to permit independent actuation of each of the three joints of the index finger. Separate actuators are used for flexion and extension, with closed-loop control of either force or position. In the future, a companion thumb exoskeleton will be developed to permit coor dinated performance of pinch. This system will be used to assess strategies for optimizing rehabilitation of pinch and reach-to-pinch following stroke. The design of the actuated index finger exoskeleton is presented here.
\end{abstract}

\section{INTRODUCTION}

$\mathrm{H}$ and impairment is a prevalent outcome for a variety of neuromuscular disorders, such as stroke. Upwards of 700,000 people in the U.S. experience a stroke each year [1]. Of these, $60-75 \%$ will live beyond one year after incidence, resulting in a current stroke population of 3 million [2-4]. Arm function is acutely impaired in a large majority of those diagnosed with stroke [5-7]. Furthermore, acute hemiparesis presages chronic hemiparesis in over $40 \%$ of the cases $[5,6]$. Chronic deficits are especially prevalent in the distal upper extremities. In fact, finger extension is the motor function most often impaired [8].

This distal limb impairment is especially problematic, because proper hand function is crucial to manual exploration and manipulation of the environment. Indeed, loss of hand function is a major source of disability in neuromuscular disorders, frequently preventing effective self-care and limiting employment opportunities. One study in the UK reported that more than half of the subjects

Manuscript received February 11, 2007. This work was supported in part by the Davee Foundation and the Coleman Foundation.

T. T. Worsnopp is a doctoral student in the Department of Mechanical Engineering of Northwestern University, Evanston, IL, 60208 USA (e-mail: greycloak@northwestern.edu).

M. A. Peshkin is a professor in the Department of Mechanical Engineering and Co-Director of the Laboratory for Intelligent Mechanical Systems, Northwestern University, Evanston, IL, 60208 USA (e-mail: peshkin@northwestern.edu).

J.E. Colgate is a professor in the Department of Mechanical Engineering and Co-Director of the Laboratory for Intelligent Mechanical Systems, Northwestern University, Evanston, IL, 60208 USA (e-mail: colgate $(0$ northwestern.edu)

D. G. Kamper is an assistant professor in the Department of Biomedical Engineering, Illinois Institute of Technology and a Research Scientist at the Rehabilitation Institute of Chicago, Chicago, IL 60611 USA (phone: 312238-1233; fax: 312-238-2208; e-mail: d-kamper@northwestern.edu). studied were dependent on others for help in the activities of daily living six months post-stroke [9].

The incidence of stroke increases with age. For men, the prevalence increases from $2.2 \%$ of the population for ages $45-54$ to $12.5 \%$ of the population for ages 75 and over [10]. As the population ages, overall prevalence will inevitably rise. Life expectancy for stroke survivors is expected to increase as well, which implies that those with chronic disabilities will require greater support.

Estimates of the number of stroke survivors returning to work vary greatly, from $3 \%-81 \%$ depending on demographics and definitions of work [11]. Recent studies, however, suggest that only $40 \%-50 \%$ of individuals are able to return to work following a stroke $[12,13]$.

An assortment of interventions has been tried in an effort to improve function or to treat the resulting peripheral alterations following stroke. Those with the most success to date tend to focus on repetitive practice. Indeed numerous studies employing the constraint-induced technique, in which focus is placed on intensive practice with the impaired arm without using the less impaired arm, have shown improvement in hand capabilities [14-16]. This supports the observations in animal models of stroke in which practice appears to be the primary factor leading to synaptogenesis and brain plasticity [17-19]. Indeed, imaging performed during constraint-induced training studies has shown evidence of cortical plasticity following the training $[20,21]$.

Unfortunately, many stroke survivors do not possess sufficient sensorimotor control to practice the desired movements. For the lower extremity, body-weight supported treadmill training has been introduced in order to address this problem $[22,23]$. As treadmill training may still be labor intensive, requiring assistance from one or more therapists for walking, robotic machines have been introduced in order to assist with this task and to hopefully make this treatment more readily available [24]. Similarly, for the upper extremity, robots have been created to assist with therapeutic training of the arm and shoulder [25-28]. Recent results describing the use of robots to promote movement of the wrist and forearm have also been encouraging [29]. It has been reported that robot-delivered sensorimotor training enhanced the motor performance of the exercised shoulder and elbow with improved functional outcome [30] and that practicing with a robot that assisted reaching movements helped the users learn how to generate smoother unaided reaching trajectories [31].

Questions remain, however, as to how best to use these robotic devices to facilitate rehabilitation. Should the device assist or resist movement? Should movement error actually be augmented, as some have suggested [32]? Should 
emphasis be placed on practice of movement of individual joints [29] or on the coordination of multiple joints?

To address these questions for the hand, an actuator is needed which can provide precise control of individual joints. Options for both force and position control are necessary to permit testing of a variety of rehabilitation strategies. Importantly, the arm should not be restricted as movement of the hand may need to be incorporated with movement of the arm, as in many activities of daily living. Imposed joint torques should be sufficiently strong to resist user input (at least $2 \mathrm{~N}-\mathrm{m}$ at the most proximal finger joint). Response time must be sufficiently fast to allow for error augmentation or reduction during the course of finger movement. Unloaded joint rotation should approach $1000 \% \mathrm{sec}$, a value we have recorded during voluntary movement in our laboratory.

A number of devices have been developed for actuating the hand. Some, such as CyberGrasp (Immersion Corporation, San Jose, CA), were intended originally to provide haptic feedback but could be employed in rehabilitation as well [33-35]. Other devices have been developed to provide user feedback in a master-slave arrangement [36, 37] or to reduce hand fatigue [38]. Finally, several devices have been described explicitly for use in hand rehabilitation, including Hand Mentor (Kinetic Muscles Inc., Tempe, AZ) and Rutgers Master II-ND [39], among others [40-44].

The majority of the developed systems do not allow for independent control of the joints. Others have insufficient torque or overly restrict arm movement. One exoskeleton which does allow independent control of finger joints has been designed for rehabilitation of occupational injuries [40]. Due to the nature of its transmission, however, response times are on the order of seconds which is too slow for our purposes.

Thus, we are developing a new device. As the hand is exceedingly complex (with more than 20 degrees-offreedom), we are focusing on the functionally important pinching movement. Control of the index finger and thumb will be provided. In this paper, the design of an actuated finger exoskeleton (AFX), intended to provide independent control of all three joints of the index finger, is described. This represents the first step in our plan to examine rehabilitation strategies for pinch and reach-to-pinch.

\section{DEVICE DESIGN}

Design of the AFX consists of three parts: mechanical exoskeleton, actuation with motors, and local control using feedback provided by sensors. These subsystems are described in the following sections.

\section{A. Exoskeleton}

The AFX has a metal (aluminum and D2 steel) exoskeleton that connects to the proximal, middle, and distal segments of the finger through pairs of rods (see Fig. 1a). Specifically, pairs of rods contact the ventral and dorsal sides of each segment of the digit to control movement of that segment about the proximal joint. Straps (not illustrated) are used to prevent motion of the finger in abduction/adduction with respect to the exoskeleton.

The AFX has three pin joints which match the metacarpophalangeal (MCP) proximal interphalangeal (PIP), and distal interphalangeal (DIP) joints of the finger. To avoid the potential difficulties associated with a remote center of rotation, each of the rotational axes of the exoskeleton is aligned with the anatomical joint of the digit. Thus, the AFX sits on the radial side of the index finger (Fig. 1). Its width profile has been reduced as much as possible $(\sim 6 \mathrm{~mm})$ to avoid interference with the thumb. The design consists of four sections that move relative to one another and are connected in series.

a)

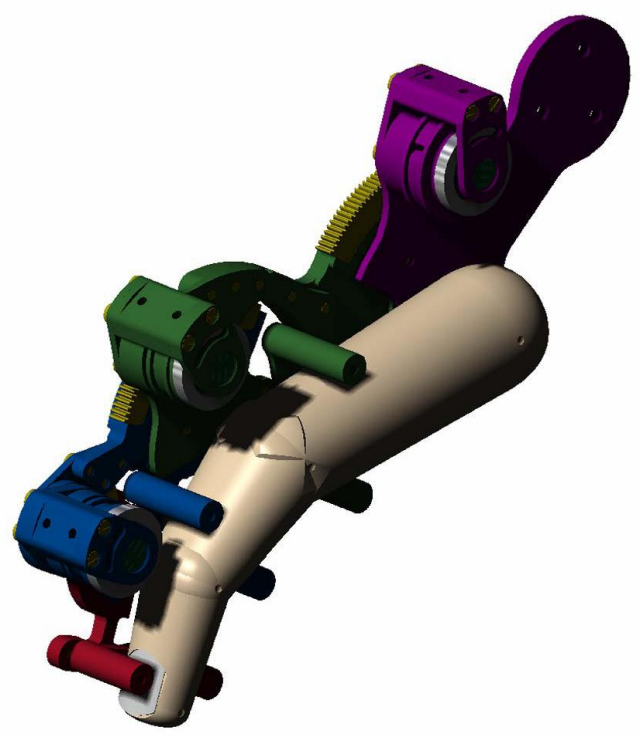

b)

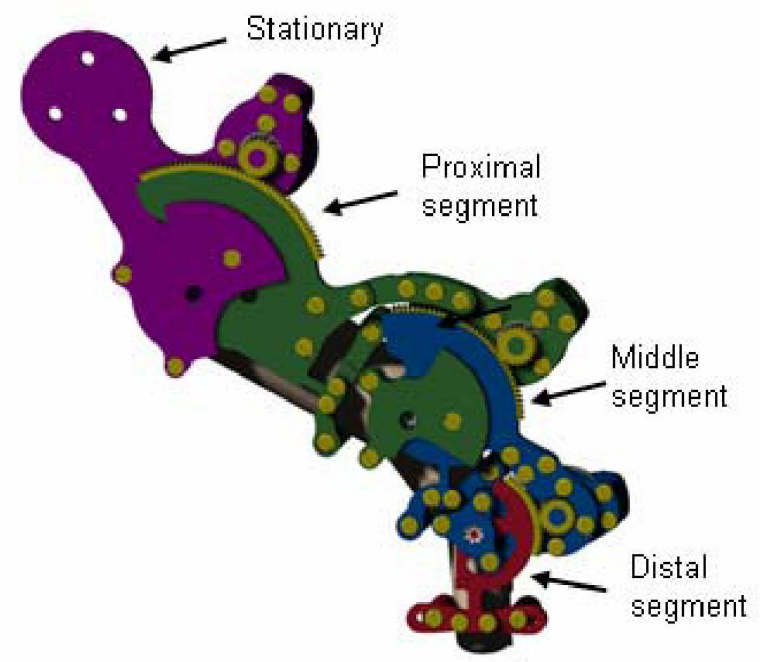

Fig. 1. SolidWorks (SolidWorks Corp., Concord, MA) drawing of AFX exoskeleton. a) Pairs of metal rods connect the AFX to each of the finger segments. b) Design consists of four sections: the most proximal ssection remains stationary with respect to the hand; the next section moves with the proximal segment; the following section moves with the middle finger segment, and the distal section moves with the distal finger segment. 
The most proximal section does not move with the finger. It is anchored to a plate secured above the forearm. Stroke survivors may have difficulty controlling wrist posture, with a tendency to assume a flexed posture not conducive to pinching or grasping. Thus, we will use a rigid splint to maintain a wrist extension angle of roughly $20^{\circ}$. The plate will be connected to the splint.

The larger gear in contact with the proximal section marks the beginning of the next section (Fig. 1b). This section contacts the proximal finger segment and rotates in correspondence with the MCP joint (see Fig. 2). The next section contacts the middle finger segment and rotates with the PIP joint. The distal section contacts the distal finger segment and rotates with the DIP joint. Smooth motion between adjacent sections is ensured by bearings. At the MCP and PIP joints, thrust bearings must be used to absorb large radial and axial loads.
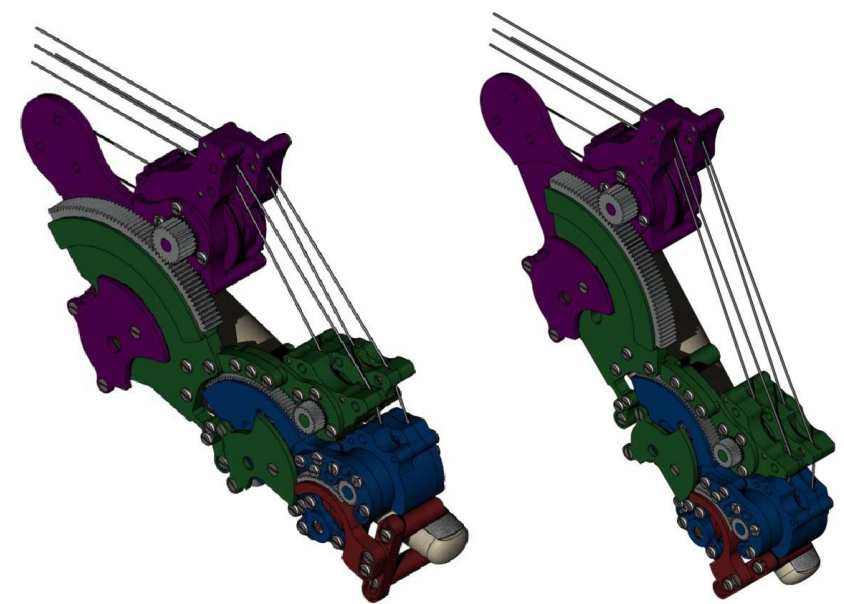

Fig. 2. Movement of the two mating proximal gears with respect to each other, producing MCP rotation (greater MCP flexion shown in right image).

Adjustment of segment lengths for different users will be performed by replacing the links between each joint of the manipulator to match the finger segment lengths (these components are easy and inexpensive to produce in different sizes). For different thicknesses of digits, the distances between each pair of contact rods can be adjusted by exchanging the component to which the contact rods are mounted for another with a different rod separation distance.

The mass of the exoskeleton that moves with the finger is $145 \mathrm{~g}$. The mass of the structure depicted (see Fig. 1) is $230 \mathrm{~g}$.

\section{B. Actuation}

To minimize the mass added to the finger, the actuators for the joints are located on the forearm. Force is transmitted to the exoskeleton through cable (Spectra, Honeywell, Colonial Heights, VA). The Spectra cable has an approximate diameter of $0.25 \mathrm{~mm}$ and is rated for $220 \mathrm{~N}$ of tension. While we have observed creep in the Spectra cable when placed under high load for days, over the course of a session we anticipate very little creep.

These cables ride along miniature cable guide pulleys attached to the exoskeleton (Fig. 3). At the intended joint, the cable is secured to a large pulley such that movement of the cable produces equivalent movement of the pulley. The pulley is press-fit onto a shaft with a small gear.

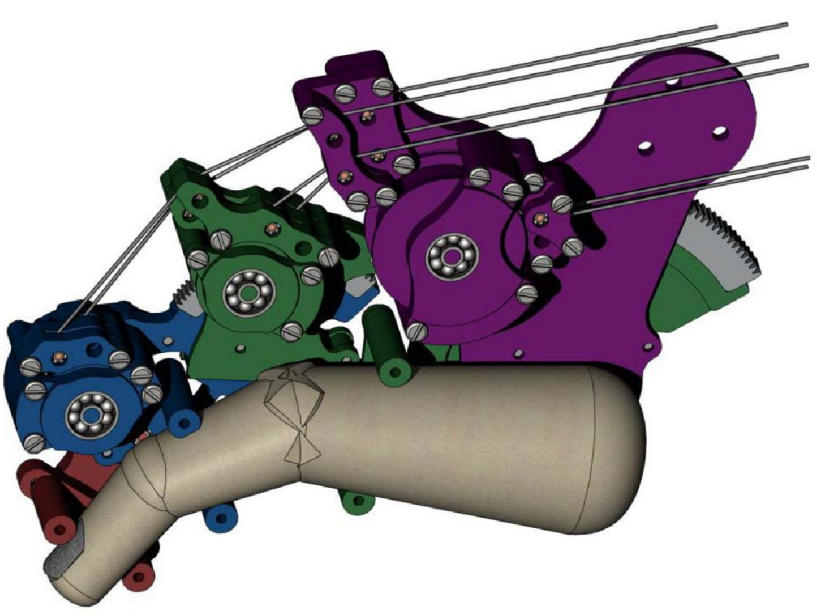

Fig. 3. View with removal of some components to expose the transmission. A pair of actuator cables connect to drive pulleys at each joint, thereby focusing power transmission at the intended joint.

In turn, the small gear transmits power to the larger gear segment on the adjacent section of the manipulator (Fig. 4). Gears with very small pitch (47 teeth per $\mathrm{cm}$ at the DIP joint, 38 teeth per $\mathrm{cm}$ at the PIP joint, and 28 teeth per $\mathrm{cm}$ at the MCP joint) are used to reduce the effects of backlash as much as possible. The gear ratios are: 3.85 at the DIP joint, 6 at the PIP joint, and 7.5 at the MCP joint.

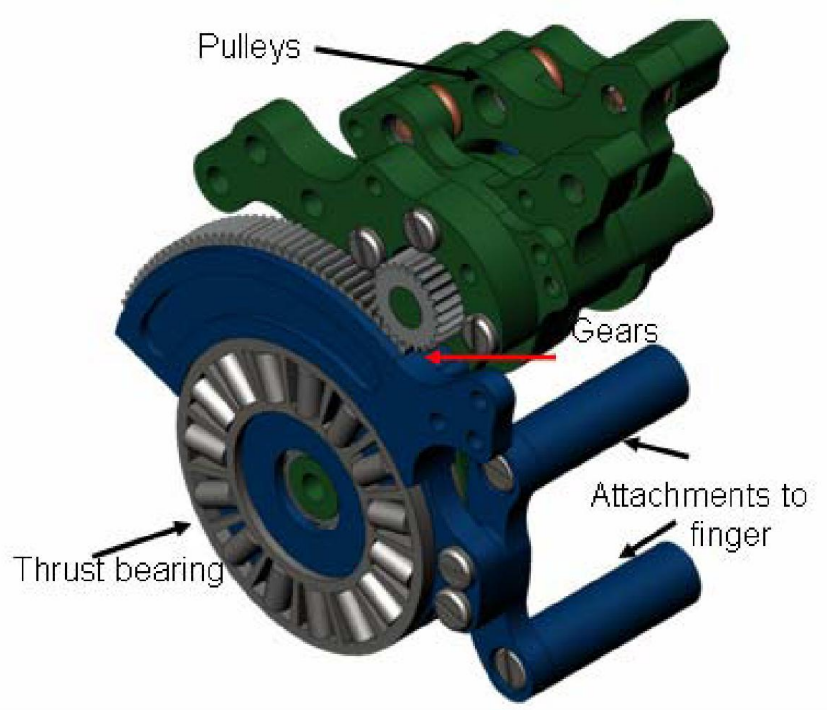

Fig. 4. Expansion of the PIP joint of the AFX. Cables from the DC motor actuators are attached to the pulleys which rotate the smaller gear. The larger gear is connected to the section of the exoskeleton which is attached to the finger segment distal to the joint (middle segment in this case).

As with muscles, the cables can only pull, they cannot push. Belt-driven or chain-driven transmissions with a single actuator are not feasible as the distance along the back 
of the digit from the base to the tip varies as the digit flexes. Thus, analogous to muscles, two cables are required for each joint: one to provide a flexion force and one to provide an extension force (see Fig. 5). Each cable is routed back to a brushless DC servomotor (AKM, Danaher Motion, Wood Dale, IL) mounted on the forearm plate. The motor controls cable length and tension. Two motors (total of 6) are used for each joint to provide the antagonistic actuation (more actuators than degrees-of-freedom). As the torque requirements for the joints are different, differently sized motors are used to minimize weight and volume (AKM13C for MCP, AKM12C for PIP, and AKM11C for DIP).

MCP abduction/adduction motion is not actuated. As most pinching movements (e.g., lateral and palmar) involve movement of the index finger in primarily the sagittal plane at neutral abduction/adduction, we do not believe that this is a fundamental limitation. Different fixed abduction angles could be accommodated by positioning of the exoskeleton with respect to the attachment on the forearm plate.

\section{Sensing and Local Control}

Joint angle will be computed using data from optical encoders attached to the DC motors. As the pulley sizes and gear ratios at each joint are fixed, motor shaft rotation can be translated into joint angle.

Sensors will also be used to measure cable tensions. As the cable is spooled from the motor, it runs over a small pulley mounted on a cantilever beam (Fig. 5). The beam is instrumented with strain gages to determine the force applied by the cable. Net cable tension is found by computing the difference between $\mathrm{T}_{\mathrm{ext}}$ and $\mathrm{T}_{\mathrm{flex}}$. The joint torque can be calculated from this value with knowledge of the pulley height above the joint and the gear ratio.

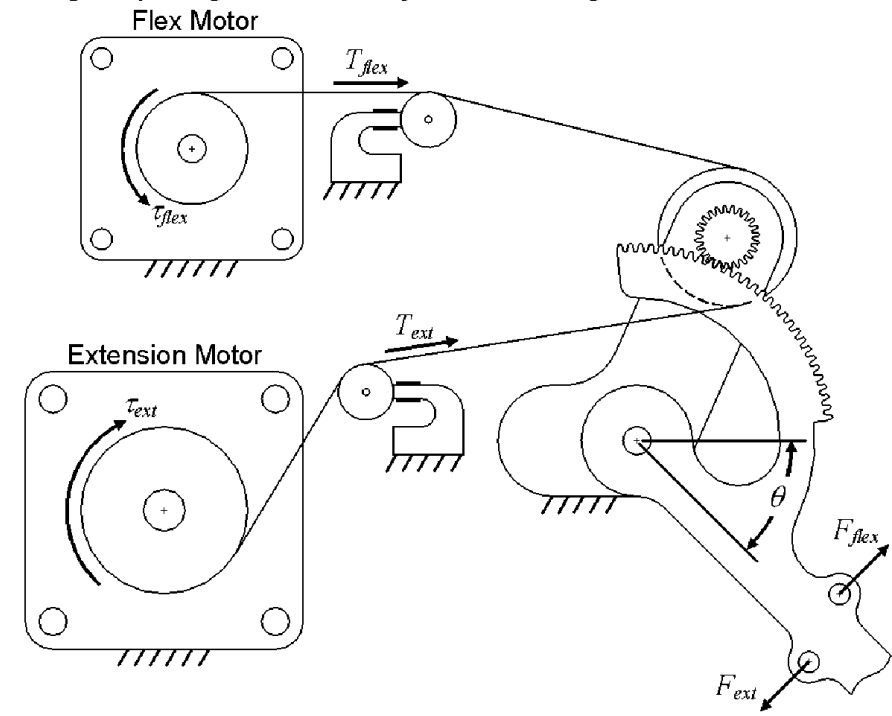

Fig. 5. Schematic of one joint of the manipulator (no friction modeled). It is assumed that frictional losses are minimal and the tension in the cable does not vary between the motor and the joint. The force is measured with tension sensors as depicted.

The torque and angle data will be used to implement local feedback control of each joint. Dependent upon the application, either control of position or control of force may be required. A position controller enables imposition of specific trajectories. A force controller is needed in order to implement virtual interactions with the user, as well as during periods when we want the manipulator to feel transparent to the user (little or no impedance). Passive backdrivability is limited by the presence of the gears and motors, but through proper control we can achieve this transparency. We will be able to implement either position or force control.

One concern of using two motors to actuate the same joint is the undesirable antagonism between the two motors. The antagonistic effects are pronounced in the AHX since the motors are only able to pull on the cables, and cannot push. At best, these two motors will perform as well as a single motor of the same size, operating directly at the joint. In order to obtain optimal performance from the two motors, we must create a controller that minimizes the antagonistic effects. Others have successfully implemented controllers to do this $[45,46]$.

Control commands must be sent to both the motor driving the load (driving motor) and the other motor (following motor). The tension in the cable attached to the following motor should only be high enough to keep the cable taut $\left(T_{\text {bias }}\right)$. The following motor uses a standard proportional-integrative-derivative (PID) controller to match the cable tension, $T_{\text {following, }}$ to the bias tension, $T_{\text {bias }}$. The driving motor also uses a PID controller to enforce the cable tension, $T_{\text {driving, }}$ which is being matched to the desired cable tension, $T_{d}+T_{\text {following }}$ (see Fig. 6). A friction model, which is based on the measured tensions and the velocities of the cables, is used to compensate for the effects of friction in reducing cable tensions at the joints.

All motors are controlled using custom software developed for the real-time operating system QNX (QNX Software Systems, Ottawa, Ontario).

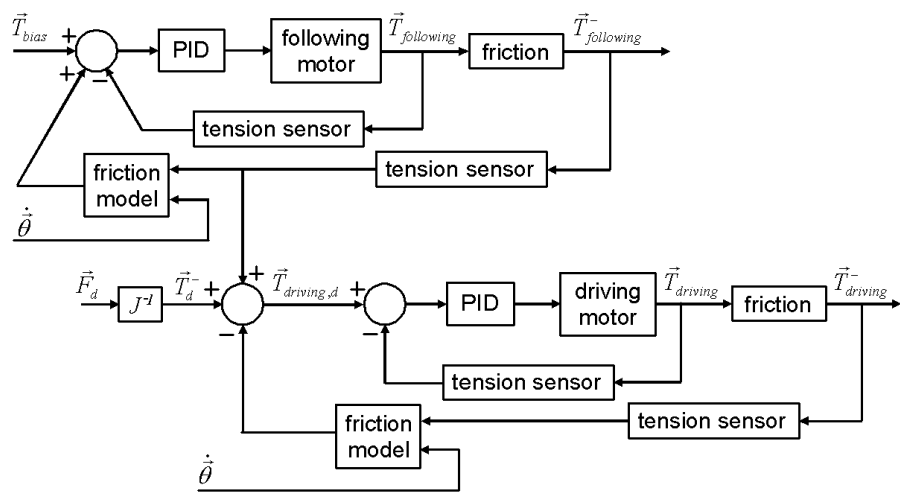

Fig. 6. Block diagram of force controller with friction.

\section{Safety and Limitations}

A number of safety mechanisms are implemented to ensure safe interaction with the individual's hand. Our design incorporates mechanical stops that limit the angular range of each joint (MCP: $-15^{\circ}-75^{\circ}$, PIP: $0^{\circ}-90^{\circ}$, and DIP: $0^{\circ}-75^{\circ}$ ). The use of an emergency-off switch provides additional safety. Software limits, monitoring both position and force, will further prevent excessive joint rotation, 
ensuring that the DIP flexion angle does not exceed that of the PIP joint, for example. With our design, differently sized motors can be used not just for the different joints, but also for the different directions at each joint. In this manner, peak motor torque can be better matched to peak voluntary subject torque and the potential for excess torque is minimized.

As with any design, limitations exist with our proposed design of AFX. For example, with our design there could be issues with backlash due to imperfect mating between the gears. We are using gears with very fine teeth, which should help minimize this problem. The weight of the actuators is significant. These actuators must move with the arm in order to maintain their relationship with the finger. A passive arm orthosis (T-WREX) [47] is available in our laboratory to help compensate for the weight of the AFX. Of course, the increased inertia will still be an issue. Finally, the abduction/adduction motion of the index finger will be constrained to a single fixed value.

\section{Simulation Results}

A static failure analysis was performed in MATLAB (MathWorks, Waltham, MA) for every component in the exoskeleton. Multiple postures were tested. The maximal cable forces that could be withstood by the exoskeleton were determined by these simulations.

Results of this analysis revealed that the bearings originally intended to be used at the MCP and PIP joints might have failed well before other components. Thus, these bearings were replaced with thrust bearings (Fig. 4) in the design. The peak joint torque that could be attained at each joint is shown in Table I. Although these values are smaller than the absolute maximum values produced by a group of neurologically intact subjects in our laboratory, they are in line with values for maximum voluntary contractions in stroke survivors.

TABLE I

AFXPARAMETERS

\begin{tabular}{lcc}
\hline \hline Joint & Peak Joint Torque & Reflected Inertia \\
\hline DIP & $0.25 \mathrm{~N}-\mathrm{m}$ & $0.006 \mathrm{~kg}$ \\
PIP & $0.75 \mathrm{~N}-\mathrm{m}$ & $0.039 \mathrm{~kg}$ \\
MCP & $2.0 \mathrm{~N}-\mathrm{m}$ & $0.783 \mathrm{~kg}$
\end{tabular}

The reflected inertias for the motors at each joint were also computed (Table I). Although this reflected inertia is fairly large, especially for MCP, the use of cable tension sensors near the motor will allow us to create a tight control loop around the motor to regulate the tension in the cables directly and mask much of the reflected inertia using the motor itself.

\section{CONCLUSIONS}

An actuated finger exoskeleton (AFX) is being developed to provide a test bed for evaluating the efficacy of different rehabilitation strategies in restoring control of pinch and reach-to-pinch movements following stroke. The
AFX will be coupled with an exoskeleton currently being designed for the thumb to permit precise, independent control of each joint of the index finger and thumb, the primary participants in pinching tasks.

Currently, the components for the exoskeleton of the AFX are being machined. All of the DC motors, amplifiers, and support electronics have been acquired. We anticipate completing the prototype and initiating performance testing within the next few months.

\section{REFERENCES}

[1] J. Broderick, T. Brott, R. Kothari, R. Miller, J. Khoury, A. Pancioli, J. Gebel, D. Mills, L. Minneci, and R. Shukla, "The Greater Cincinnati/Northern Kentucky Stroke Study: preliminary first-ever and total incidence rates of stroke among blacks," Stroke, vol. 29, pp. 415-21, 1998.

[2] R. Bonita, Steward, A, Beaglehole, R, "International trends in stroke mortality: 1970-1985," Stroke, vol. 21, pp. 989-992, 1990.

[3] J. P. Broderick, S. J. Phillips, J. P. Whisnant, W. M. OFallon, and E. J. Bergstralh, "Incidence rates of stroke in the eighties: the end of the decline in stroke?," Stroke, vol. 20, pp. 577-582, 1989.

[4] American Heart Association, "Heart and Stroke Facts," American Heart Association, Dallas, TX 1991.

[5] C. S. Gray, J. M. French, D. Bates, N. E. Cartlidge, O. F. James, and G. Venables, "Motor recovery following acute stroke," Age Ageing, vol. 19, pp. 179-84, 1990.

[6] H. Nakayama, H. S. Jorgensen, H. O. Raaschou, and T. S. Olsen, "Recovery of upper extremity function in stroke patients: The Copenhagen Stroke Study," Arch Phys Med Rehabil, vol. 75, pp. 394-398, 1994.

[7] V. M. Parker, D. T. Wade, and R. Langton Hewer, "Loss of arm function after stroke: measurement, frequency, and recovery.," Int Rehabil Med, vol. 8, pp. 69-73, 1986.

[8] C. A. Trombly, "Stroke," in Occupational Therapy for Physical Dysfunction, C. A. Trombly, Ed. Baltimore: Williams and Wilkins, 1989 , pp. $454-471$

[9] D. Wade, "The epidemiologically based needs assessment reviews. Volume I," in Health Care Needs Assessment, vol. Volume I, J. Raftery, Ed., 1994, pp. 111-255.

[10] American Heart Association, "Heart Disease and Stroke Statistics - 2004 Update," American Heart Association 2004

[11] R. M. Black-Schaffer and L. Lemieux, "Vocational outcome after stroke," Top Stroke Rehabil, vol. 1, pp. 74-86, 1994.

[12] M. A. Wozniak, S. J. Kittner, T. R. Price, J. R. Hebel, M. A. Sloan, and J. F. Gardner, "Stroke location is not associated with return to work after first ischemic stroke," Stroke, vol. 30, pp. 2568-73, 1999.

[13] M. Vestling, B. Tufvesson, and S. Iwarsson, "Indicators for return to work after stroke and the importance of work for subjective well-being and life satisfaction," J Rehabil Med, vol. 35, pp. 127-31, 2003.

[14] S. L. Wolf, S. Blanton, H. Baer, J. Breshears, and A. J. Butler, "Repetitive task practice: a critical review of constraint-induced movement therapy in stroke," Neurologist, vol. 8, pp. 325-38, 2002.

[15] C. J. Winstein, D. K. Rose, S. M. Tan, R. Lewthwaite, H. C. Chui, and S. P. Azen, "A randomized controlled comparison of upper-extremity rehabilitation strategies in acute stroke: A pilot study of immediate and long-term outcomes," Arch Phys Med Rehabil, vol. 85, pp. 620-8, 2004.

[16] S. J. Page, S. Sisto, P. Levine, and R. E. McGrath, "Efficacy of modified constraint-induced movement therapy in chronic stroke: a single-blinded randomized controlled trial," Arch Phys Med Rehabil, vol. 85, pp. 14-8, 2004.

[17] T. A. Jones, C. J. Chu, L. A. Grande, and A. D. Gregory, "Motor skills training enhances lesion-induced structural plasticity in the motor cortex of adult rats," J Neurosci, vol. 19, pp. 10153-63, 1999. 
[18] J. A. Kleim, T. A. Jones, and T. Schallert, "Motor enrichment and the induction of plasticity before or after brain injury," Neurochem Res, vol. 28, pp. 1757-69, 2003.

[19] T. A. Jones and T. Schallert, "Use-dependent growth of pyramidal neurons after neocortical damage," $J$ Neurosci, vol. 14 , pp. $2140-52,1994$.

[20] J. Liepert, H. Bauder, W. H. R. Miltner, E. Taub, and C. Weiller, "Treatment-induced cortical reorganization after stroke in humans," Stroke, vol. 31, pp. 1210-1216, 2000.

[21] J. Liepert, W. H. R. Miltner, H. Bauder, M. Sommer, C. Dettmers, E. Taub, and C. Weiller, "Motor cortex plasticity during constraint-induced movement therapy in stroke patients," Neurosci Letters, vol. 250, pp. 5-8, 1998.

[22] H. Barbeau and M. Visintin, "Optimal outcomes obtained with body-weight support combined with treadmill training in stroke subjects," Arch Phys Med Rehabil, vol. 84, pp. 1458-65, 2003.

[23] S. Hesse and C. Werner, "Partial body weight supported treadmill training for gait recovery following stroke," $A d v$ Neurol, vol. 92, pp. 423-8, 2003.

[24] P. Winchester and R. Querry, "Robotic orthoses for body weight-supported treadmill training," Phys Med Rehabil Clin N $A m$, vol. 17 , pp. 159-72, 2006 .

[25] L. R. Macclellan, D. D. Bradham, J. Whitall, B. Volpe, P. D. Wilson, J. Ohlhoff, C. Meister, N. Hogan, H. I. Krebs, and C. T. Bever, Jr., "Robotic upper-limb neurorehabilitation in chronic stroke patients," J Rehabil Res Dev, vol. 42, pp. 717-22, 2005.

[26] M. Ferraro, J. J. Palazzolo, J. Krol, H. I. Krebs, N. Hogan, and B. T. Volpe, "Robot-aided sensorimotor arm training improves outcome in patients with chronic stroke," Neurology, vol. 61, pp. 1604-7, 2003.

[27] D. J. Reinkensmeyer, L. E. Kahn, M. Averbuch, A. McKennaCole, B. D. Schmit, and W. Z. Rymer, "Understanding and treating arm movement impairment after chronic brain injury: progress with the ARM guide," J Rehabil Res Dev, vol. 37, pp. 653-62, 2000.

[28] P. S. Lum, C. G. Burgar, P. C. Shor, M. Majmundar, and M. Van der Loos, "Robot-assisted movement training compared with conventional therapy techniques for the rehabilitation of upperlimb motor function after stroke," Arch Phys Med Rehabil, vol. 83, pp. 952-9, 2002.

[29] S. Hesse, C. Werner, M. Pohl, S. Rueckriem, J. Mehrholz, and M. L. Lingnau, "Computerized arm training improves the motor control of the severely affected arm after stroke: a single-blinded randomized trial in two centers," Stroke, vol. 36, pp. 1960-6, 2005.

[30] B. T. Volpe, H. I. Krebs, N. Hogan, O. L. Edelstein, C. Diels, and $\mathrm{M}$. Aisen, "A novel approach to stroke rehabilitation: robotaided sensorimotor stimulation," Neurology, vol. 54, pp. 193844,2000 .

[31] L.E. Kahn, M. L. Zygman, W. Z. Rymer, and D. J. Reinkensmeyer, "Effect of robot-assisted and unassisted exercise on functional reaching in chronic hemiparesis," presented at $23 \mathrm{rd}$ Annual IEEE Engineering in Medicine and Biology Conference, Istanbul, Turkey, 2001.

[32] J. L. Patton, M. E. Stoykov, M. Kovic, and F. A. Mussa-Ivaldi, "Evaluation of robotic training forces that either enhance or reduce error in chronic hemiparetic stroke survivors," Exp Brain Res, vol. 168, pp. 368-83, 2006.

[33] M. Bergamasco, "Force replication to the human operator: the development of arm and hand exoskeletons as haptic interfaces," presented at 7th International Symposium of Robotics Research, Munich, Germany, 1995.

[34] B. H. Choi and H. R. Choi, "SKK hand master hand exoskeleton driven by ultrasonic motors," presented at IEEE/RSJ International Conference on Intelligent Robots and Systems, Takamatsu, Japan, 2000.

[35] U. Mali and M. Munih, "HIFE-haptic interface for finger exercise," IEEE/ASME Transactions on Mechatronics, vol. 11, pp. 93-102, 2006.

[36] S. Nakagawara, I. Kawabuchi, H. Kajimoto, N. Kawakami, and $\mathrm{S}$. Tachi, "An Encounter-type multi-fingered master hand using circuitous joints," presented at IEEE International Conference on Robotics and Automation, Barcelona, Spain, 2005.
[37] B. M. Jau, "Dexterous telemanipulation with four fingered hand system," presented at International Conference on Robotics and Automation, Tokyo, Japan, 1995.

[38] B. L. Shields, J. A. Main, S. W. Peterson, and A. M. Strauss, "An anthropomorphic hand exoskeleton to prevent astronaut hand fatigue during extravehicular activities," IEEE Trans Syst Man Cybern A Syst Hum, vol. 27, pp. 668-673, 1997.

[39] D. Jack, R. Boian, A. S. Merians, M. Tremaine, G. C. Burdea, S. V. Adamovich, M. Recce, and H. Poizner, "Virtual realityenhanced stroke rehabilitation," IEEE Trans Neural Syst Rehabil Eng, vol. 9, pp. 308-18, 2001.

[40] A. Wege and G. Hommel, "Development and control of a hand exoskeleton for rehabilitation of hand injuries, " presented at IEEE/RSJ International Conference on Intelligent Robots and Systems, Edmonton, Canada, 2005.

[41] I. Sarakoglou, N. G. Tsagarakis, and D. G. Caldwell, "Occupational and physical therapy using a hand exoskeleton based exerciser," presented at IEEE/RSJ International Conference on Intelligent Robots and Systems, Sendai, Japan, 2004.

[42] M. DiCicco, L. Lucas, and Y. Matsuoka, "Strategies for an EMG-controlled orthotic exoskeleton for the hand," presented at IEEE International Conference on Robotics and Automation, New Orleans, USA, 2004.

[43] C. D. Takahashi, V. H. Der-Yeghiaian, and S. C. Cramer, "A robotic device for hand therapy after stroke," presented at IEEE International Conference on Rehabilitation Robotics, Chicago, IL, 2005.

[44] T. Kline, D. G. Kamper, and B. D. Schmit, "Control system for pneumatically controlled glove to assist in grasp activities," presented at IEEE International Conference on Rehabilitation Robotics, Chicago, IL, 2005.

[45] S. C. Jacobsen, H. Ko, and E. K. Iversen, "Control Strategies for Tendon-Driven Manipulators," IEEE Control Systems Magazine, pp. 23-28, 1990.

[46] S. Ma and M. Watanabe, "Minimum-time control of coupled tendon-driven manipulators," Advanced Robotics, vol. 15, pp. 409-427, 2001.

[47] R. Sanchez, D. J. Reinkensmeyer, P. Shah, J. Liu, S. Rao, R. N. Smith, S. C. Cramer, T. Rahman, and J. Bobrow, "Monitoring functional arm movement for home-based therapy after stroke," presented at 26th Annual International Conference of IEEE EMBS, San Francisco, CA, 2004. 